\title{
Influence of sleep-onset time on the development of 18-month-old infants: Japan
}

\section{Children's cohort study.}

\author{
Akiko Iemura M.D ${ }^{\mathrm{a}, \mathrm{b}^{*}}$, Mizue Iwasaki PhD ${ }^{\mathrm{a}, \mathrm{b}^{*}}$, Noriko Yamakawa M.D ${ }^{\mathrm{a}, \mathrm{c}}$, Kiyotaka \\ Tomiwa M.D ${ }^{\mathrm{a}, \mathrm{d}}$,Yoko Anji ${ }^{\mathrm{a}, \mathrm{e}}$, Yoichi Sakakihara M.D ${ }^{\mathrm{a}, \mathrm{e}}$, Tatsuyuki Kakuma,D.Sc. ${ }^{\mathrm{f}}$ \\ Shinichiro Nagamitsu M.D ${ }^{\mathrm{b}}$, and Toyojiro Matsuishi, M.D ${ }^{\mathrm{a}, \mathrm{b}}$ \\ aJapan Children's Study Group, Research Institute of Science and Technology for \\ Society, Japan Science and Technology Agency, Tokyo, Japan \\ ${ }^{\mathrm{b}}$ Department of Pediatrics and Child Health, Kurume University School of Medicine,
}

Fukuoka, Japan

${ }^{\mathrm{c} C l i n i c a l}$ Research Institute, Mie-chuo Medical Center, National Hospital Organization,

Mie, Japan

${ }^{\mathrm{d}}$ Graduate School of Medicine; Kyoto University; Kyoto, Japan

${ }^{\mathrm{e} O c h a n o m i z u}$ University; Tokyo,Japan

${ }^{\mathrm{f}}$ Centre for Bio-Statistics, Kurume University School of Medicine; Fukuoka, Japan.

*Akiko Iemura and Mizue Iwasaki contributed equally as first co-authors

(C) 2015. This manuscript version is made available under the Elsevier user license http://www.elsevier.com/open-access/userlicense/1.0/ 
Iemura A. et al. 2

25 text pages and 3 tables, and 3 figures 
*Corresponding author:

Toyojiro Matsuishi, M.D.

Department of Pediatrics and Child Health, Kurume University School of Medicine, 67

Asahi-machi, KurumeCity, Fukuoka 830-0011, Japan

Tel: +81-942-31-7565, Fax: +81-942-38-1792;

E-mail: toyojiro@st-mary-med.or.jp 


\begin{abstract}
OBJECTIVE: We report here the influence of sleep patterns on the development of infants in Japan. A total of 479 infants were registered in two different Japanese cities.

Direct neurological observations were performed by licensed pediatric

neurologists.METHOD: We designed a prospective cohort study and identified the sleep factors of children showing atypical development. The Kinder Infant Developmental Scale (KIDS) was used to evaluate the infant developmental quotient (DQ); we also applied a neurobehavioral screening battery. Neurobehavioral observations in 18-month-old infants were designed to check all developmental categories within the three areas of motor function, language, and social function. Based on the observations, each infant was classified as having "atypical development"or "typical development".
\end{abstract}

RESULT: We found that later sleep onset time (> 22:00 h), and longer naps during the day each had significant positive correlations with atypical development patterns in 18-month-old infants. For each hour the infant sleep-onset time extended past 22:00 h, the infants showed worse neurodevelopmental outcomes, at an odds ratio increase of 2.944.CONCLUSION: Although our results may be confounded by sleep problems resulting from pre-existing developmental disabilities, we can safely conclude that 
Iemura A. et al. 5

appropriate sleeping habits are important for healthy development in 18-month-old

infants.

Key words: cohort study, sleeping habits, social development 


\section{Introduction}

During the period of early infancy, sleep patterns begin to change dramatically

[1-3].Sleep deprivation and other sleep problems in children caused by factors such as late sleep onset and curtailed nocturnal sleep are a major public health concern in Japan and worldwide $[4,5]$.Sleep problems in adolescents and adults have also increased worldwide. Despite these sleep-related health concerns, relevant epidemiological studies have been limited, with very few being comprehensive enough to show a correlation between sleep status and mental, cognitive, behavioral, and/or physical health; further, only a few of these have been prospective cohort studies [6-9].We therefore designed a prospective study to investigate the relationship between sleep status and social competence in Japanese infants [10]. This study was based on the understanding that incomplete acquisition of social competence underlies social problems in children, resulting in irritability, school refusal, mild developmental disorders, adolescent delinquency, social withdrawal, infant care anxiety, and child abuse [11, 12]. While some manuscripts report that the development of sociality in children is affected by individual factors such as temperament and inborn growth/development properties, others emphasize environmental factors such as family and social environment, behaviors of parents, and the mother-child relationship [13-15]. The development of 
sociality in children is known to be affected by a combination of biological and psychosocial factors; however, its relation to sleep patterns remains insufficiently researched.

Previous cohort studies seeking to identify the underlying cause of specific problems in children have not included direct developmental and behavioral observations in collaboration with pediatric neurologists or psychologists. Recently, late onset and shorter sleep periods have been tentatively linked to the cognitive and behavioral functions of infants and children $[16,17]$. Children with autism spectrum disorder (ASD) or attention deficit hyperactivity disorder (ADHD) have shown higher rates of nonstandard sleep factors [18-24].However, only a few studies have been designed as prospective cohort studies, and most of these were based on questionnaires. We therefore designed a cohort study that relied on direct evaluation of behavior, including minor neurological signs, to evaluate the relationship between sleep patterns and child development. We hypothesized that sleep patterns, especially late sleep-onset time resulting in a shorter night's sleep, would show deleterious effects in the development of 18-month-old infants.

\section{Methods}




\section{Participants}

The Japan Children's Study (JCS) is a unique prospective cohort study that commenced in 2005 and was conducted by the JCS research group at two study sites in Japan. The JCS was designed to assess factors affecting development in children, in particular those affecting social skills (children's sociability) [10].Infants born between 2004 and 2005 together with their parents were selected from two sampling areas, Osaka and Mie City, to participate in this study.The developmental ages of prospective subjects in this project were 4, 9, 18, and 30 months of age. We chose the 18-month-old infants to enroll in the final study because from this age we requested that the parents maintain a sleep diary designed by us [25].Sleep is a phenomenon thought to involve a mixture of biological and psychosocial factors [3]. In analyzing the extensive questionnaires and observational data necessary for such cohort studies, we thus examined factors that affect the development of sociality in 18-month-old infants using various sleep variables as criteria. This study used data from the JCS project covering 479 families,including mothers and children, where serial observations and follow-up was possible for 4-, 9-, and 18 month-old infants. Children whose mothers completed the questionnaires at baseline and continued to participate in observations at 9 and 18 months of age (403 infants, $84.1 \%$ ) were eligible to stay in the study. The follow-up rate 
of 18-month-old infants was $86 \%$ in Osaka and $92 \%$ in Mie City. Direct neurological observations were performed by licensed Japanese pediatric neurologists.

\section{Exclusion criteria}

Infants born prematurely (gestation period less than 36 weeks) and those with multiple congenital anomalies (more than 4 anomalies), chromosomal abnormalities, or congenital intrauterine TORCH infections (toxoplasma, rubella, cytomegalovirus, herpes infection, etc.) were excluded from this study because infants with these condition are known to have underlying mental and behavioral abnormalities and skewed development.

\section{Measurements}

\section{Sleep schedules}

In the present study, we used the Japan Children's Study Sleep Questionnaire (JCSSQ) to evaluate infant sleep patterns at the age of 18 months. JCSSQ is a unique questionnaire that allows the parameters of "wake time", "bed time", "number of nights waking", "nap time" and so on to be addressed for weekdays, Saturdays, and Sundays using three separate timelines in a form that is simple and easy for caregivers to fill in (Fig 1) [25,26]. Therefore, we evaluated the sleep diary data for weekdays, Saturday and Sunday representatively within a 2-week period. 
We previously confirmed the efficacy of our novel sleep diary [25]. In our previous study, we found that infant sleep is largely influenced by the parents sleep hygiene [26]. Fathers work hard until Saturday in most cities in Japan. Therefore, Friday is more like a weekday than the weekend.Parents were requested to complete the JCSSQ.

\section{Neurobehavioral observations}

Neurobehavioral observations in 18-month-old infants were designed to check all developmental categories within this area $[10,27]$. Neurological examinations included gross and fine motor functions such as muscle tone, standing and walking posture, fine motor skills when playing with toys, building with blocks, scribbling with a pencil, and pointing out body parts such as eye, mouth, nose, and ear. Infants were also asked to point out specific items such as a dog, doll, cup, fish, scissors or a picture book. Social functions consisted of gazing at objects and eye-to-eye contact with the mother. We also used direct observation of important items in MCHAT[28]: namely (original item numbers in parentheses following question), Does your child take an interest in other children? (2), Does your child ever use his/her index finger to point, to indicate interest in something? (7) Does your child imitate you? (13) Does your child respond to his/her name when you called? (14) and If you point at the toy across the room, does your child 
look at it? (15).Language functions included perception and expression of language, and the number of words spoken.For neurobehavioral observations, development can be separated into motor development, cognitive behavior development, and social cognitive development. Based on observations in these three areas, each 18-month-old infant was classified as having atypical development or typical development. Infants in whom all three areas were observed as normal and well-balanced were classified into the "typical development" group, whereas those in whom deviation or an imbalance developmentamong the three areas was recognized were classified into the "atypical development" group. These neurobehavioral observations took about 45 minutes

\section{[27].Questionnaires}

In addition to JCSSQ, we used the following questionnaires and data to identify factors influencing child development, as determined by the Kinder Infant Development.Scale (KIDS) [29]: Evaluation of Environmental Stimulation Short version (EESS) [30],AutisticBehaviorSymptoms [32], Stressful Life Event [32],Child Temperament (Original Scales and Items) [33,34], and physical information including height, body weight, and head circumference. KIDS has nine categories including Physical motor (physical motor ability), Manipulation (fine motor ability), Receptive language and Expressive language (verbal ability), Concept (cognitive abilities), Social relationships 
with other childrenand Social relationships with adults (social behavior), Discipline (development of self-control) and Feeding (eating habits).Temperament has 4 subcategories including approach to / withdrawal from to new situations and negative emotionality and frustration tolerance and attention span and persistence.

Information on parents' age, level of education, and annual family income, as well as the children's sex, birth weight, birth order, and gestational age was obtained from a questionnaire completed by mothers [10].

\section{Statistical analysis}

First, we used Student's t-test to compare the average sleeping items recorded in the developmental profiles of infants evaluated as having "atypical development" or "typical development" according to neurobehavioral observations. Next, we examined the relationship between significant and marginally significant sleeping items using the generalized additive model. Data analyses were conducted using this binary outcome as the response variable. Because there were many risk factors, the tree-based data mining technique was used to screen out unimportant factors. Non-linear relationships between outcomes and selected risk factors were then identified using the generalized additive models $(*)$. After fitting the model, partial residual plots with smoothed spline curves were created for graphic representation of the non-linear effects of risk factors on 
outcome. A final logistic regression model for multiple risk factors was then constructed.

Spline functions were introduced to approximate the non-linear effects of risk factors.

Then, using the Random Forest and Stochastic Gradient Boosting methods, we selected

variables from the development profiles using related indexes including response

variables not in the development profile including basic information, sleeping items

assessed at 18 months of age, media, temperament, autism, developmental age,

childhood maltreatment, mother/father attributes, family economy, child care

environment, perinatal period, sleeping items at four months of age, sleeping items at

nine months of age, and whether the mother smoked. Finally, we applied the

multivariate additive logistic model to the variables data, deleted the insignificant

variables, and then built a multivariate model. Data mining was carried out using

TreeNet $(* *)$ software, while the $\mathrm{R}$ package was used for constructing the generalized

additive models. The statistical package SAS was used for all other statistical analyses.

(* Simon N. Wood (2006) Generalized Additive Models: An Introduction with R. New

York, Chapman \& Hall/CRC) (** TreeNet, Salford Systems, San Diego, Calfornia,

USA).

Ethics

This study protocol was approved by the Ethical Review Committee of the 
Research Institute of Science and Technologyfor Society, Japan Science and

Technology Agency and theEthical Review Committee of each institute of the JCS

researchgroups, based on the Guidelines ConcerningEpidemiologicalResearch of the

Ministry of Education, Culture, Sports, Scienceand Technology and the Ministry of

Health Labor and Welfare in Japan [10]. The study protocol was also approved by the

Ethics Committee of the Kurume University School of Medicine.

\section{Results}

\section{Neurodevelopmental assessments at 18 months of age}

Serial observations were done in 403 infants from 479 families (follow-up rate;

$84.1 \%$ ). Of these, 300 infants were evaluated as having typical development, and 80

were evaluated as having atypical development. We diagnosed 19 children as having deviated development, and 61 children as having imbalanced development, based on the neurobehavioral observations. Then, we confirmed similar tendencies in sleep items and other background items. Finally, we performed statistical analysis on the combined group of deviated / imbalanced development as "atypical. The remaining 23 infants were unevaluated due to incomplete information.

\section{KIDS}


Using KIDS B, we evaluated DQ scores in atypical children $(\mathrm{n}=80)$ and typical children ( $\mathrm{n}=300)$ and found no significant difference between the scores of atypical children (100.05 \pm 10.5$)$ and typical children (109.0 \pm 9.4$)$

\section{Comparison of sleep-related variables in 18-month-old infants (Table 1)}

Examination of sleep onset times showed that on weekdays and Sundays, infants in the typical group fell asleep more quickly than those in the atypical group $(P=0.0075$, $P=0.029)$. For the Saturday daytime sleep, infants in the atypical group had a shorter morning daytime nap, afternoon daytime nap, and total daytime nap than those in the typical development group $(P=0.0188, P=0.0358$, and $P=0.006$, respectively $)$. For nocturnal sleep on weekdays, Saturdays and Sundays, infants in the typical group had a longer sleep time than those in the atypical group $(P=0.0035, P=0.0075$, and $P=$ 0.0025 , respectively), while there was a significant difference in sleep efficiency-the ratio of time actually slept to time spent in bed, $\times 100 \%$ - on Saturdays $(P=0.0079)$. The sleep-related variables producing significant differences between the two groups were similar among weekdays, Saturdays, and Sundays.

\section{Relationship among data collected in the development profiles of 18-month-old infants and significant sleeping variables using the generalized additive model} Univariate analysis showed that infants in the typical development group had an 
earlier sleep-onset time, shorter daytime nap time, and spent a longer time in bed compared to those in the atypical development group (Table 2).

Identification of variables related to the data collected in the development profiles of 18-month-old infantsusing methods of Random Forest and Stochastic Gradient

\section{Boosting methods}

The tree-based data-mining procedure identified 17 risk factors that contributed to adverse sleep times in infants. Using the generalized additive model, 8 of these 17 factors remained significant: In the order of the most significant, Discipline ( $P=$ 0.0008),Expressive language ( $P=0.001)$, Receptive language $(P=0.001)$, Head circumference $(P=0.007)$, Sleep efficiency (Saturdays) $(P=0.009)$, Concept $(P=$ 0.002),Sleep-onset time (weekdays) $(P=0.007)$, and Approach to new situations ( $P=$ 0.011) (Table 3).

The final logistic model reflects non-linear effects of risk factors on the outcome

Among the sleep items, sleep efficiency (Saturdays) and sleep-onset time (weekdays) proved the most important components for typical development. The effect of sleep efficiency (Saturdays) was linear. When the estimated parameter increased by one point, the odds for the child to be classified in the atypical group (for each of the items listed above) changed to $0.927(P=0.0075)$ (Fig 2). The non-linear effect of 
sleep-onset time on the outcome can be graphically viewed by a partial residual plot

with a smoothed spline curve. There were no effects of risk factors for sleep-onset times prior to 22:00. However, after 22:00, and for each hour thereafter, the odds of the child being in the atypical group increased to $2.944(P=0.003)$ (Fig 3).

\section{Discussion}

The present study showed that the development of 18-month-old infants is affected by their sleeping habits. Later sleep-onset time (> 22:00 h), and longer naps during the day showed significant associations with atypical development patterns in 18-month-old infants.In our cohort study, all infants were assessed for development and classified, not only by the KIDS B, but also by licensed Japanese neuro-pediatricians through observations. This direct examination for infant's development leads to a more reliable relationship between infant development and their sleep habits.

The assessment of development profiles by pediatric neurologists revealed that more infants with a late sleep-onset time, short sleeping hours during the night, and reduced sleep efficiency on Saturdays were classified as having atypical development than infants with healthy sleep habits. Further, when infant sleep-onset time extended one hour past 22:00 h, the infants showed worse neurodevelopmental outcomes (odds 
ratio increase of 2.944-fold); this finding demonstrated the possibility that sleep and development in "atypical development" may reveal only an association ora causal relationship between sleep habits and development. The 18-month period after birth is very important in childhood development, especially for the development of fine motor skills or coordinated motion and social cognition. In addition, it is important to consider both environmental effects and the child's innate capabilities as factors that affect childhood development [15-17]. In this report, we discuss findings that demonstrate an association between sleep and development. First, we discuss the involvement of sleep as an environmental factor affecting development. During the first 18 months after birth, children are considered to be markedly affected by the rhythm of sleeping and by the lifestyles of their caregivers. Touchette et al. reported that such a tendency starts in 4-month-old infants where sleep-onset time is delayed on weekends, even without the infants' strong preference for a delayed sleep time [23].In the present study, the tendency for the sleep-onset time of 18-month-old infants to be delayed on weekends but not on weekdays was found in both typical and atypical infants. Further, bedtime on Sundays was significantly later in atypical infants than in typical infants. Taking these findings together, it can be considered that for atypical infants, the sleeping time on both weekday and Sunday nights is shorter than that of typical infants. The significantly 
longer napping time on Saturday by atypical infants is considered to be compensation for reduced sleeping time during the night. While napping time on Saturdays is longer in these infants, the efficiency of sleep on Saturdays was shown to decrease. It is therefore possible that continued late bedtimes and short sleeping hours for a long period of time has an adverse effect on development processes in infants, suggesting that caregivers recognize that their own lifestyle could be a factor influencing the development of their children. In light of our findings, we suggest that caregivers be encouraged to show an active interest in their children's sleep during early childhood by, for example, checking sleep status during health checkups.

While sleep patterns appear to affect development, there are also known effects of innate developmental problems on sleep patterns in children. For instance, ASD, developmental retardation and ADHD are well known to cause sleep problems in children [18-24].In addition, there is a relationship between going to bed late and behavioral problems in children [15-17].Touchette et al. described that shortened sleep duration, especially before the age of 41 months, is associated with hyperactivity / impulsivity and lower cognitive performance at school [35].In the present study, it is likely that infants with ASD and ADHD were classified as having atypical development based on neurobehavioral observations. It is therefore possible that delayed sleep-onset 
time and short sleeping hours in these infants resulted from pre-existing developmental problems.

Head circumference, which is a clinical index of brain capacity, reflects actual brain volume, and in early childhood, variations in head circumference are proportional to brain weight and volume. This study revealed that the head circumference of atypical infants, which in this study possibly includes infants with ASD, was significantly larger than that of typical infants. This finding is consistent with recent studies indicating that ASD is associated with macrocephaly[36-41].

Our findings, together with those of other studies, suggest that sleeping problems could be an index allowing the early recognition of children with behavioral problems, such as ASD. Other variables showing a statistically significant difference between typical and atypical infants include parenting, conception, language comprehension, and language expression.

From the perspective of relationships, our association of poor sleeping habits in 18-month-old infants with atypical development likely resulted both from infants having developmental problems being unable to establish sleeping habits, and from infants with poor sleeping habits due to inappropriate parenting behavior showing atypical development. The contribution of environment is likely represented, at least in 
part, by the demonstrated difference between typical and atypical infants in the score for approach to new situations (based on the child's temperament scale). Temperament problems in children are closely associated with parenting stress and anxiety in caregivers [33, 34]. In early childhood, developmental retardation is associated with avoidance of novel stimulation and a negative temperament[33], and parenting stress in mothers is enhanced when their children show temperamental characteristics that are difficult to deal with[33,34]. It is reported that as sleeping hours during the night increase, the score of contiguity increases[42, 43]. These findings lead to the notion that developmental problems in infants together with inappropriate parenting behavior caused by parenting stress lead to problematic sleeping habits in infants. It has also been reported that parasympathetic hypoactivity and elevated heart rate are more frequent in Japanese children at the age of five or six when nocturnal sleeping hours are reduced[44].

This study has two major limitations. One is that we could not further specify the diagnosis of "atypical development", which may include children with ASD, ADHD, and other developmental disabilities as well as normal infants with atypical development. This was because the JCScohort study was finished before the final follow-up was completed and the second study comprised a relatively small sample size 
of the groups.Another limitation was that we could not use the objective sleep tools of actigraphy or polysomnography because it was not feasible to use the actigraphs in such a large cohort study. Sleep efficiency may be not so accurate, even though we previously reported that the sleep efficiency by using sleep log significantly correlated with actigraph findings $[45,46]$.

In conclusion, we directly examined all infants at 18 months of age in a cohort study, and could found a significant association between their development and sleep habits.Though sleep habits in infants are influenced by both their caregivers' lifestyles and pre-existing developmental problems, we can safely conclude that appropriate sleeping habits are important for healthy development in 18-month-old infants. 


\section{Contributors}

Mizue Iwasaki, Akiko Iemura, collected the data and wrote this manuscript, Noriko

Yamakawaperformed and directed the Mie district study, Kiyotaka Tomiwa performed the Osaka cohort study, Yoko Anji and Yoichi Sakakihara designed the method for direct neurological examination, including examination of soft neurological signs of 18-month-old infants, and analyzed the samples. Shinichiro Nagamitsu did statistical analysis and contributed to writing the manuscript. Tatsuyuki Kakuma performed the statistical analyses and constructed the statistical model. Toyojiro Matsuishi is a reader of the sleep cohort study and provided input into the study design and manuscript preparation. All the authors contributed to this paper and approved its submission.

\section{Acknowledgments}

We thank and are deeply indebted to the participating children's parents and the administrators of the Japan Children's Study. This study is based on the mission-type research project conducted by the RISTEX (Research Institute of Science and Technology for Society) and the Japan Science and Technology Agency. 


\section{APPENDICES \\ Japan Children's Study Group:}

Chairman: Zentaro Yamagata (Department of Health Sciences, School of Medicine,

University ofYamanashi), Hideaki Koizumi (Advanced Research Laboratory, Hitachi,

Ltd.)Participating Researchers: Yoko Anji, Yuka Shiotani, Mizue Iwasaki, Aya Kutsuki,

Misa Kuroki,Naho Ichikawa, Tomoyo Morita, Haruka Koike, Yusuke Morito, Shunyue

Cheng, Hiraku Ishida, Hisakazu Yanaka, Daisuke Tanaka, Kumiko Namba, Tamami

Fukushi, Hiroshi Toyoda, Shihoko Kimura-Ohba, Akiko Sawada (Research Institute of Science and Technology for Society, Japan Science and Technology Agency),

Kevin K. F. Wong (Department of Anesthesia and Critical Care,Massachusetts General Hospital), Yoichi Sakakihara (Department of Child Care and Education, Ochanomizu University), Hideo Kawaguchi (Advanced Research Laboratory, Hitachi, Ltd.), Toyojiro Matsuishi (Department of Pediatrics and Child Health, Kurume University), Shunya Sogon (The Graduate Divisiton of the faculty of Human Relations, Kyoto Koka Women's University), Kiyotaka Tomiwa, Tomonari Awaya, Sigeyuki Matuzawa (Graduate School of Medicine, Kyoto University), Shoji Itakura (Graduate School of Letters, Kyoto University), Masako Okada (Koka City Educational Research Center), Yoshihiro Komada (Department of Pediatric and Developmental 
Science, Mie University Graduate School of Medicine Institute of Molecular and

Experimental Medicine), Hatsumi Yamamoto, Noriko Yamakawa, Motoki Bonno

(Clinical Research Institute, Mie-chuo Medical Center, National Hospital Organization),

Mariko Y. Momoi, Takanori Yamagata, Hirosato Shiokawa (Department of Pediatrics.,

Jichi Medical University), Norihiro Sadato, DaisukeN Saito (National Institute for

Physiological Sciences, National Institutes of Natural Sciences),Hitoshi Uchiyama

(Matsue Co-medical College), Tadahiko Maeda, Tohru Ozaki (The Institute of

Statistical Mathematics, Research Organization of Information and Systems), Tamiko

Ogura(Graduate School of Humanities, Kobe University), Hiroko Ikeda (National

Epilepsy CenterShizuoka Institute of Epilepsy and Neurological Disorder), Koichi

Negayama (Graduate School ofHuman Sciences, Waseda University), Kayako

Nakagawa (Graduate School of Engineering, OsakaUniversity), Kanehisa Morimoto

(Graduate School of Medicine, Osaka University), Tokie Anme(Graduate School of

Comprehensive Human Sciences, University of Tsukuba), Katsutoshi Kobayashi

(Center for Education and Society, Tottori University), Tatsuya Koeda, Toshitaka

Tamaru, Ayumi Seki, Shinako Terakawa, Ariko Takeuchi (Faculty of Regional

Sciences, Tottori University), Yukuo Konishi (Department of Infants' Brain \&Cognitive

Development, Tokyo Women's MedicalUniversity), Osamu Sakura (Interfaculty 
Iemura A. et al. 26

Initiative in Information Studies, The University of Tokyo),Masatoshi Kawai (Institute

for Education, Mukogawa Women's University), Sonoko Egami (Hokkaido University

of Education), Takahiro Hoshino (Graduate School of Economics, NagoyaUniversity),

Yuko Yato (College of Letters, Ritsumeikan University). 


\section{References}

[1] Acebo C, Sadeh A, Seifer R, Tzischinsky O, Hafer A,Carskadon MA. Sleep/Wake patterns derived from activity monitoring and maternal report for healthy 1 - to 5-year-old children. Sleep 2005;28:1568-77.

[2] Sadeh A, Mindell JA, Luedtke K, Wiegand B. Sleep and sleep ecology in the first 3 years: a web-based study. J Sleep Res 2009;18:60-73.

[3] Sadeh A, Tikotzky L, Scher A. Parenting and infant sleep. Sleep Med Rev 2010;14: 89-96.

[4] Kohyama J, Mindell JA, Sadeh A. Sleep characteristics of young children in Japan: Internet study and comparison with other Asian countries. Pediatr Int 2011; 53:649-55.

[5] Mindell JA, Sadeh A, Kohyama J, How TH. Parental behaviors and sleep outcomes in infants and toddlers: a cross-cultural comparison. Sleep Med 2010;11:393-9.

[6] Gregory AM, Caspi A, Moffitt TE, Poulton R. Sleep Problems in Childhood Predict Neuropsychological Functioning in Adolescence. Pediatrics 2009;123:1171-6. 
[7] Iglowstein I, Jenni OG, Molinari L, Largo RH. Sleep duration from infancy to adolescence: reference values and generational trends. Pediatrics 2003;111:302-7.

[8] Crosby B, LeBourgeois MK, Harsh J. Racial differences in reported napping and nocturnal sleep in 2- to 8-year-old children. Pediatrics 2005;115:225-32.

[9] Spruyt K, Aitken RJ, So K, Charlton M, Adamson TM, Horne RS. Relationship between sleep/wake patterns, temperament and overall development in term infants over the first year of life. Early Hum Dev 2008;84:289-96.

[10] Yamagata Z, Maeda T, Anme T, Sadato N, Japan Children's Study Group .Overview of the Japan Children's Study 2004-2009; Cohortstudy of early childhood development. J Epidemiol 2010;20 Suppl 2:S397-403.

[11] Chen X, French DC. Children's social competence in cultural context. Annu Rev Psychol 2008;59: 591-616.

[12] Hediger ML, Overpeck MD, Ruan WJ, Troendle JF. Birthweight and gestational age effects on motor and social development. Paediatr Perinat Epidemiol 2002;16:33-46.

[13] Friedman HS. Long-term relations of personality and health: 
dynamisms,mechanisms, tropisms. J Pers 2000;68:1089-107.

[14] Anme T, Shinohara R, Sugisawa Y, Tong L, Tanaka E, Watanabe T, et al. Interaction ratingscale (IRS) as an evidence-basedpractical index of Children's Social Skills and Parenting. J Epidemiol 2010;20 Suppl 2:S419-26.

[15] Tomisaki E, Tanaka E, Shinohara R, Sugisawa Y, Tong L,Hirano M, et al.ALongitudinal Study on SocialCompetenceDevelopment and Sleeping Habits. J Epidemiol 2010;20 Suppl 2:S472-5.

[16] Largo RH, Hunziker UA. A developmental approach to the management of children with sleep disturbances in the first three years of life. Eur J Pediatr 1984;142:170-3.

[17] Hiscock H, Canterford L, Ukoumunne OC, Wake M. Adverse associations of sleep problems in Australian preschoolers: national population study. Pediatrics 2007;119:86-93.

[18] Schreck KA, Mulick JA, Smith AF. Sleep problems as possible predictors of intensified symptoms of autism. Res Dev Disabil 2004;25:57-66.

[19] Liu X, Hubbard JA, Fabes RA, Adam JB. Sleep disturbances and correlates 
ofchildren with autism spectrum disorders. Child Psychiatry Hum Dev 2006;37:

$179-91$.

[20] Kotagal S, Broomall E. Sleep in children with autism spectrum disorder. Pediatr Neurol 2012;47:242-51.

[21] Cohen-Zion M, Ancoli-Israel S. Sleep in children with attention-deficit hyperactivity disorder (ADHD): a review of naturalistic and stimulant intervention studies. Sleep Med Rev 2004;8:379-402.

[22] Goraya JS, Cruz M, Valencia I, Kaleyias J, Khurana DS, Hardison HH, et al. Sleep study abnormalities in children with attention deficit hyperactivity disorder. Pediatr Neurol 2009;40:42-6.

[23] Touchette E, Côté SM, Petit D, Liu X, Boivin M, Falissard B, et al.Short nighttime sleep-duration and hyperactivity trajectories in early childhood. Pediatrics 2009; 124:e985-93.

[24] Matsuoka M, Nagamitsu S, Iwasaki M, Iemura A, Yamashita Y, Maeda M, et al. High incidence of sleep problems in children with developmental disorders: results of a questionnaire survey in a Japanese elementary school. Brain Dev 
2014;36:35-44.

[25] Iwasaki M, Iemura A, Oyama T, Matsuishi T. A Novel Subjective Sleep

Assessment tool for healthy elementary school children in Japan. J Epidemiol 2010;20 Suppl 2:S476-81.

[26]Iwata S, Iwata O, Iemura A, Iwasaki M, Matsuishi T. Determinants of sleep patterns in healthy Japanese 5-year-old children. Int JDev Neurosci 2011;29:57-62.

[27] Anji Y, Sakakihara Y, Konishi Y, Tomiwa K. Development of a new-behavioral test battery for the assessment of infant sociability. Paper presented at the International Meeting of the Psychometric Society 2007, (Abstracts, P. 172).

[28]Robins DL, Fein D, Barton ML. Modified Checklist for Autism in Toddlers (M-CHAT). Self-published; 1999

[29] Miyake K, Ohmura M, Takashima M, Yamauchi S, Hashimoto K. Kinder infant development scale. Manual: Hattatsu Kagaku Kenkyu Kyoiku Center, Tokyo. 1989 (in Japanese).

[30] Anme T, Segal UA. Implications for the development of children in over 11 hoursof centre-based care. Child Care Health Dev 2004;30:345-52. 
[31]Ishii T, Takahashi O. The epidemiology of autistic children in Toyota, Japan: Prevalence. Jpn J Child Adolesc Psychiatry 1983;24:311-21.

[32] Shiokawa H. Development of the life event questionnaire for parents: Its use and reliability data(in Japanese). Jichi Med Unive J 2007;30:165-72.

[33] Ogura T, Itakura S, Egami S, Kutuski A, Kubo K. Development of social cognition in infancy (4): Influence of temperament(in Japanese). Proceedings of the 70th Conference of the Japanese Psychological Association. Kyushu Univ, Fukuoka 2006:1173.

[34] Spruyt K, Aitken RJ, So K, Charlton M, Adamson TM, Horne RS. Relationship between sleep/wake patterns, temperament and overall development in term infants over the first year of life. Early Hum Dev 2008;84:289-96.

[35] Touchette E, Petit D, Tremblay RE, Montplaisir JY. Risk factors and consequences of early childhood dyssomnias: New perspectives. Sleep Med Rev 2009; $13: 355-61$.

[36] Torrey EF, Dhavale D, Lawlor JP, Yolken RH. Autism and head circumference inthe first year of life. Biol Psychiatry 2004;56:892-4. 
[37] Dissanayake C, Bui QM, Huggins R, Loesch DZ. Growth in stature and head circumference in high-functioning autism and Asperger disorder during the first 3 years of life. Dev Psychopathol 2006;18:381 -93.

[38] Dawson G, Munson J, Webb SJ, Nalty T, Abbott R, Toth K. Rate of head growth decelerates and symptoms worsen in the second year of life in autism. Biol Psychiatry 2007;61:458-64.

[39] Mraz KD, Green J, Dumont-Mathieu T, Makin S, Fein D. Correlates of head circumference growth in infants later diagnosed with autism spectrum disorders. J Child Neurol2007;22:700-13.

[40] Fukumoto A, Hashimoto T, Ito H, Nishimura M, Tsuda Y, Miyazaki M, et al. Growth of head circumference in autistic infants during the first year of life. $\mathbf{J}$ Autism Dev Disord2008;38:411-8.

[41] Hazlett HC, Poe M, Gerig G, Smith RG, Provenzale J, Ross A, et al. Magnetic resonance imaging and head circumference study of brain size in autism: birth through age 2 years. Arch Gen Psychiatry 2005; 62:1366-76.

[42] Yokomaku A, Misao K, Omoto F, Yamagishi R, Tanaka K, Kohyama J, et al. A 
study of the association between sleep habits and problematic behaviors in preschool children. Chronobiol Int 2008;25:549-64.

[43] Kohyama J. The possible long-term effects of early-life circadian rhythm disturbance on social behavior. Expert Rev Neurother 2014;14:745-55.

[44] Sampei M, Dakeishi M, Wood DC, Iwata T, Murata K. Spontaneous awakening from nocturnal sleep and cardiac autonomic function in preschool children. Auton Neurosci 2007;133:170-4.

[45]Matsuishi T. Evaluation of various parameters in Japanese cohort children's study. Symposium on "Suku-Suku”Cohort (Japan Children's Study) For the future of Children Proceedings 2009:31-2 (in Japanese).

[46] Iwasaki M, Iwata S, Iemura A, Yamashita N, Tomino Y, Anme T, et al. Utility of subjective sleep assessment tools for healthy preschool children: a comparative study between sleep logs, questionnaires, and actigraphy. J Epidemiol 2010;20:143-9.

Figure Legends. 
Fig. 1. The Japan Children's Study Sleep Questionnaire(JCSSQ).

Waking time and sleep-onset time were recorded separately for weekdays, Saturdays, and Sundays.Parents were requested to complete the JCSSQ.

Fig. 2. Sleep efficiency (weekend)

The effect is linear for Sleep efficiency (Saturday): namely, when the estimated parameter decreases, odds for the child to be an atypical one are $0.927 .(p=0.0075)$

Fig. 3. Sleep-onset time (weekdays) $>22$

For the sleep-onset time (weekdays), no effect presents in prior to 22:00; after 22:00, for each hour getting later, odds of the child to be an atypical one increase 2.9435 .

$(\mathrm{p}=0.003)$ 

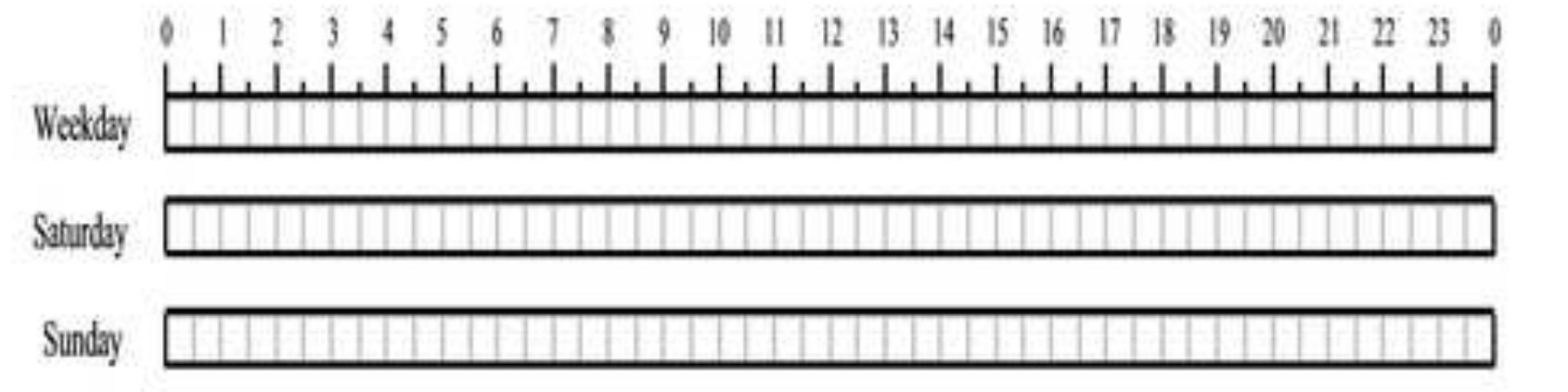

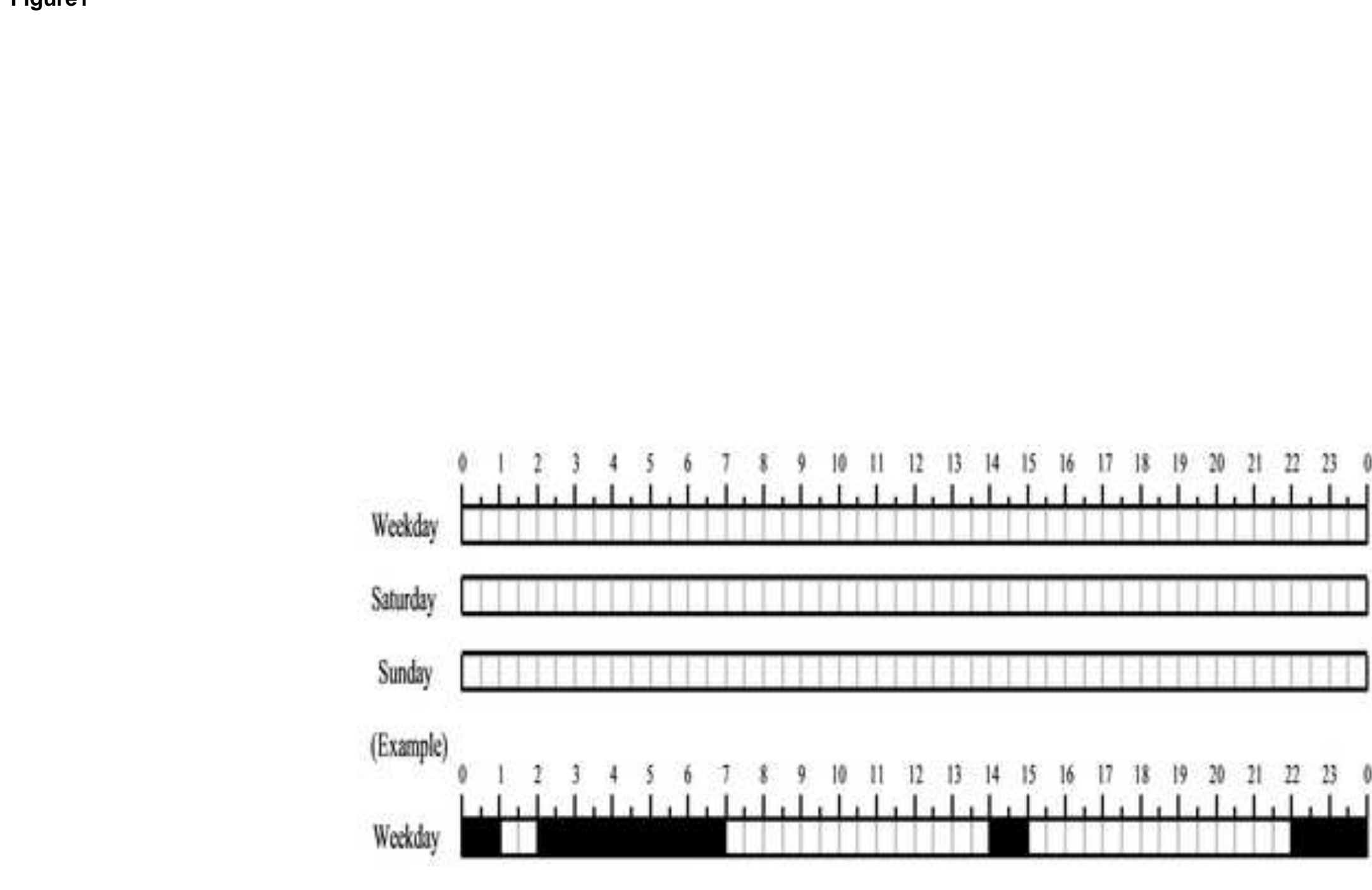

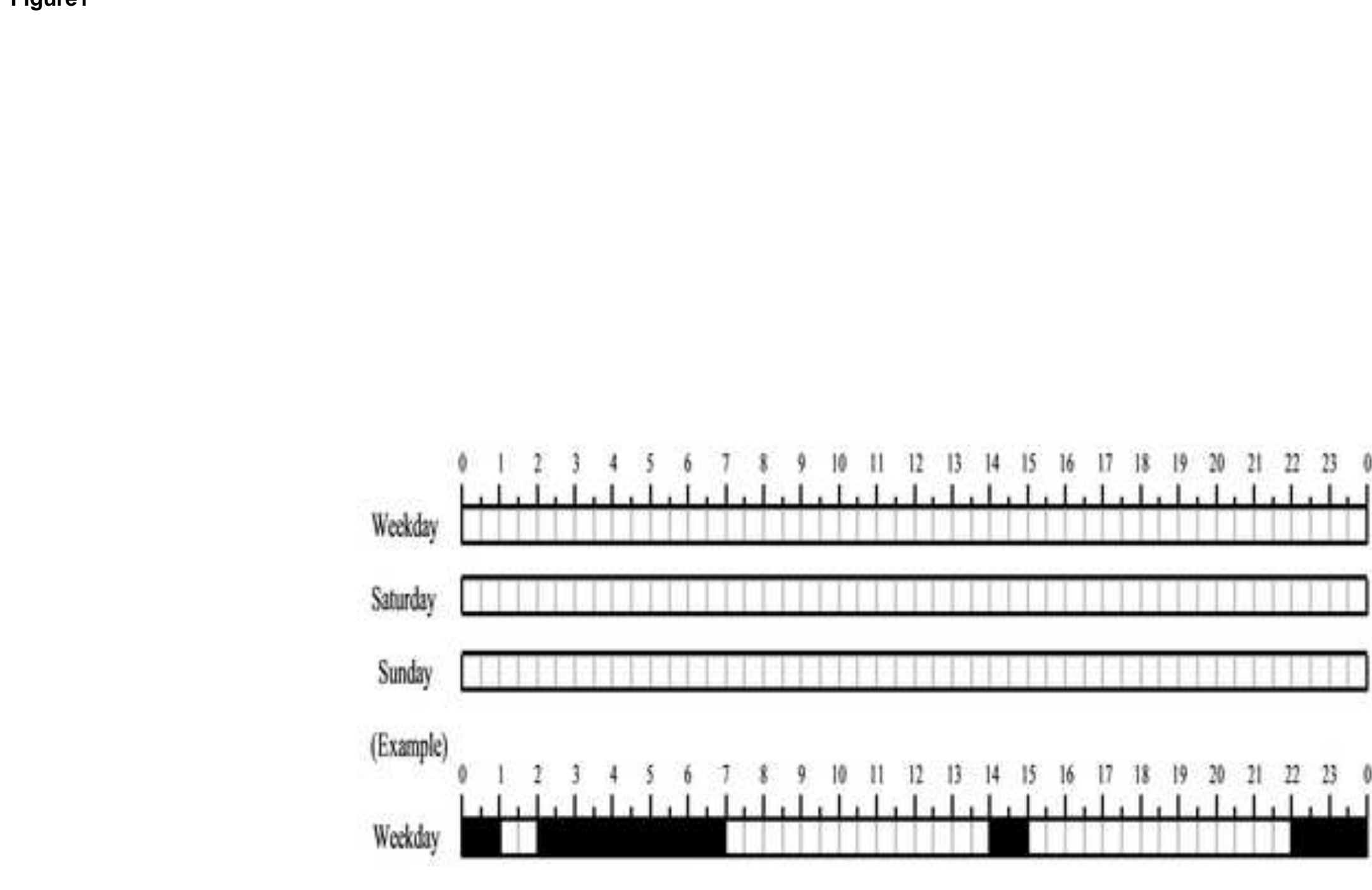

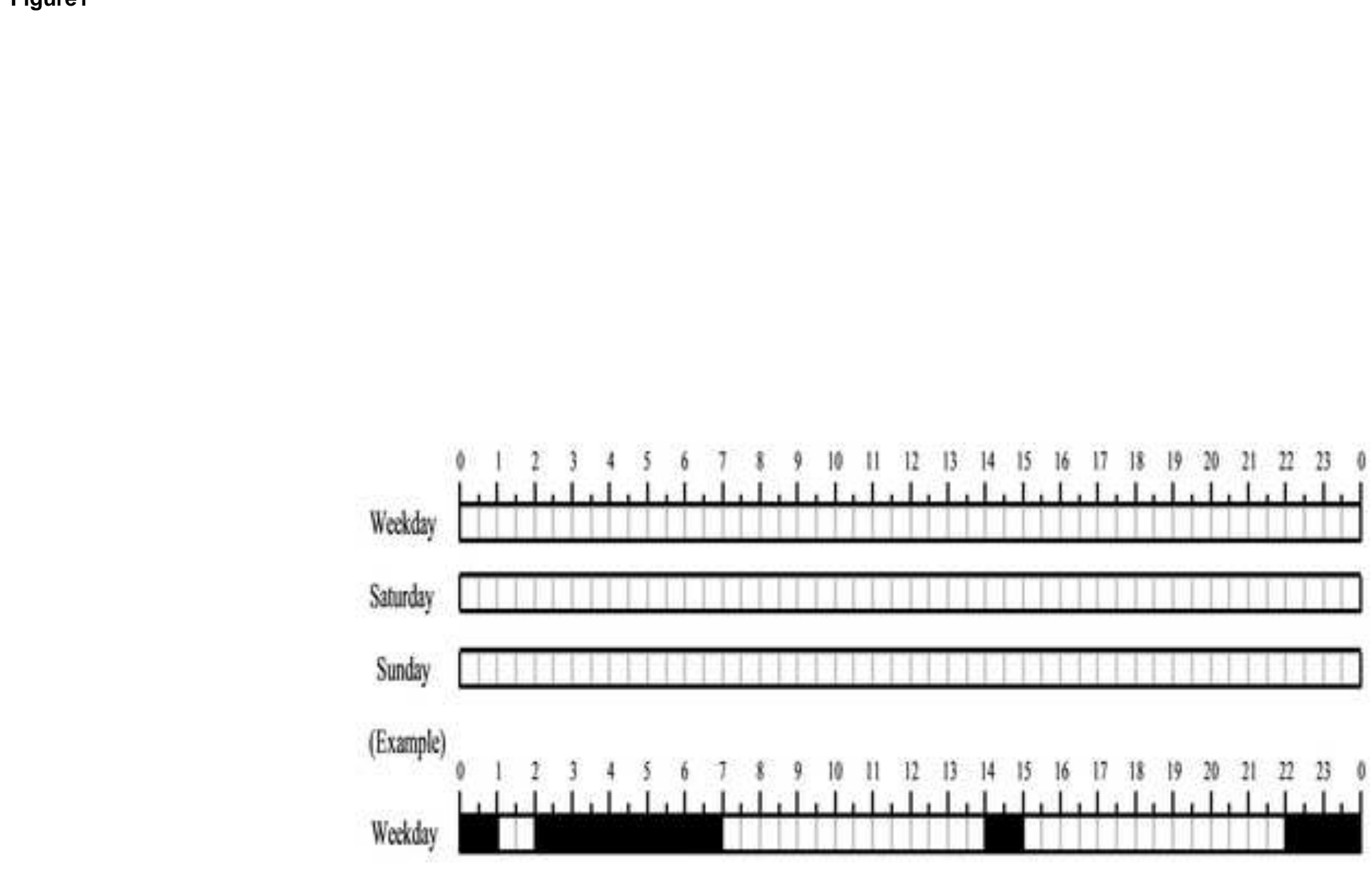

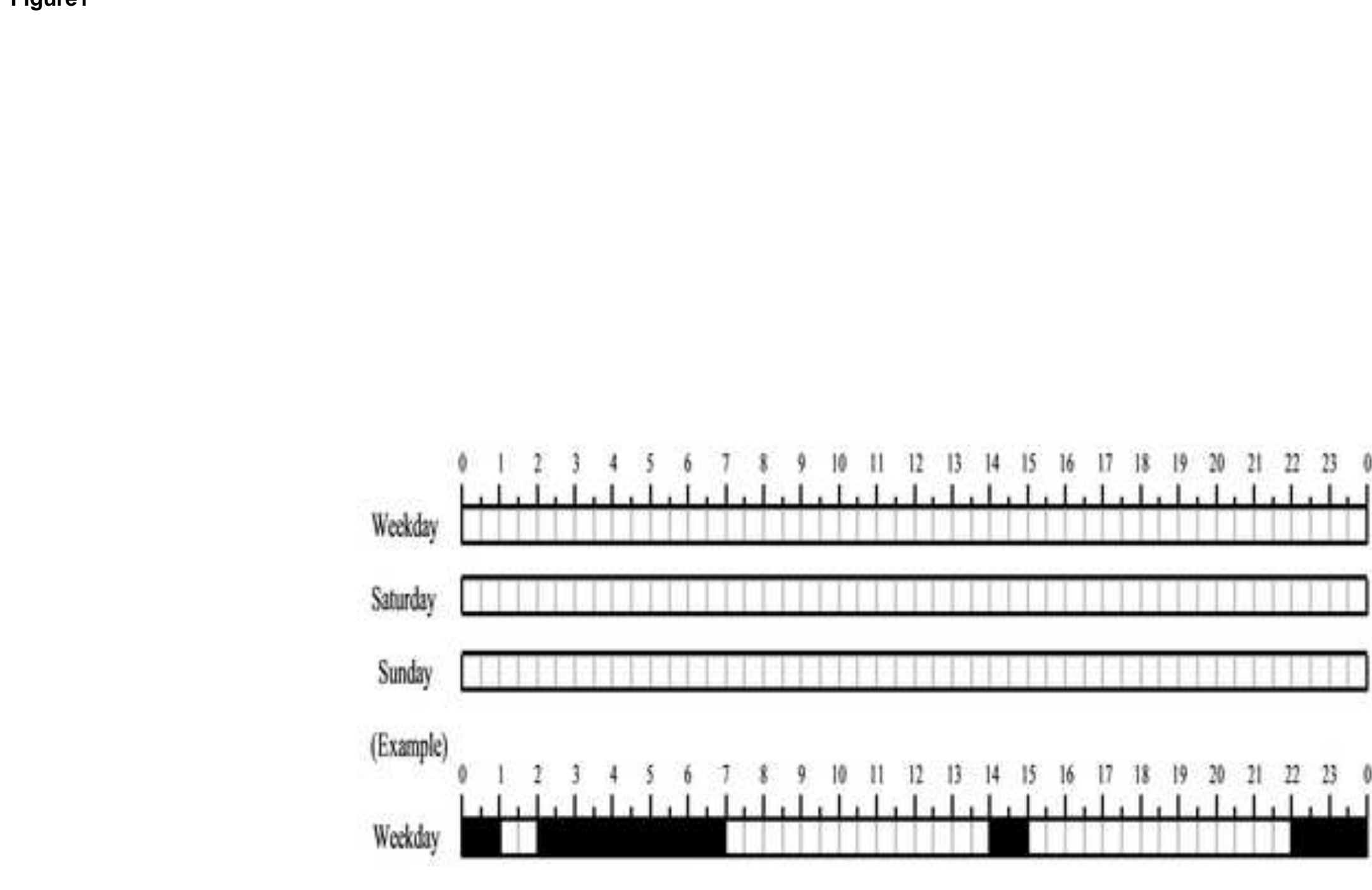

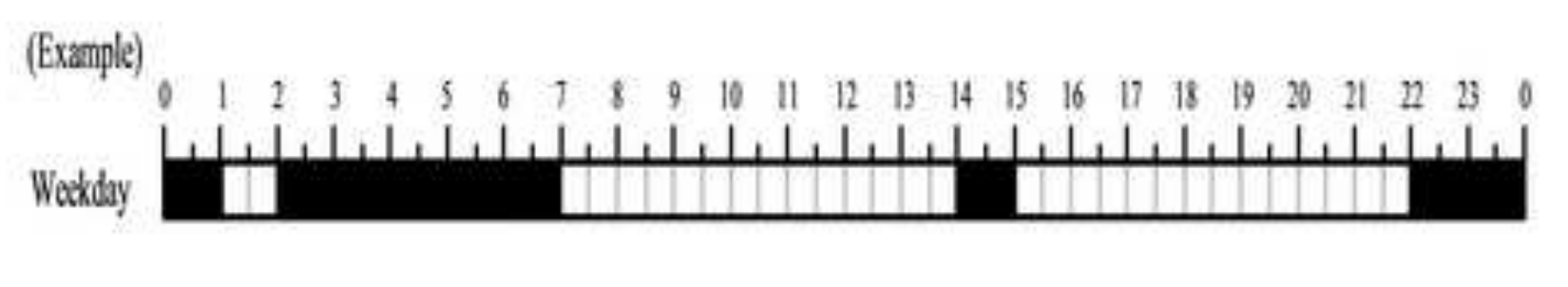

.

ن. . . 


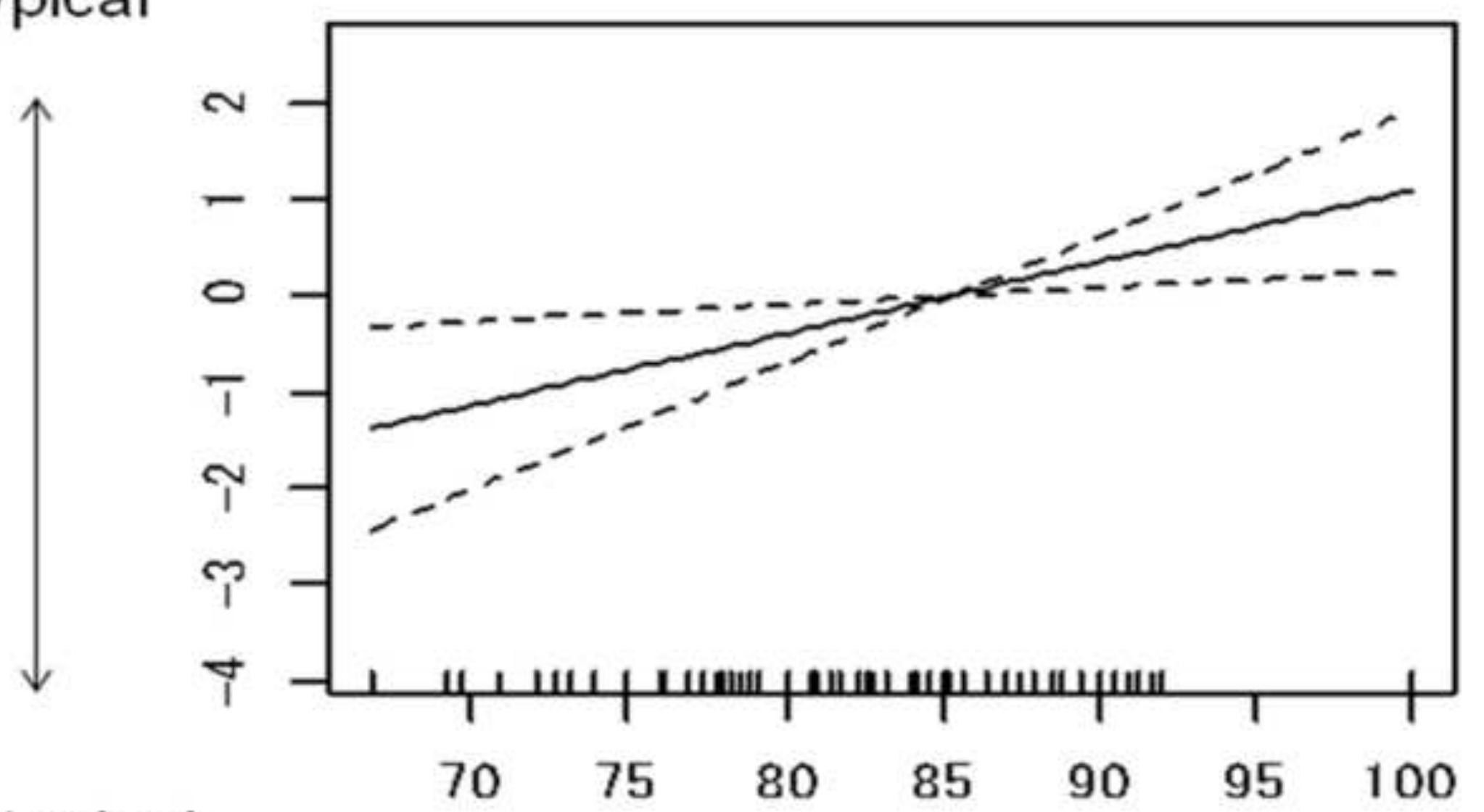

Atypical $\begin{array}{lllllll}70 & 75 & 80 & 85 & 90 & 95 & 100\end{array}$

\section{Typical}

I

$$
\begin{aligned}
& \text { Typical }
\end{aligned}
$$

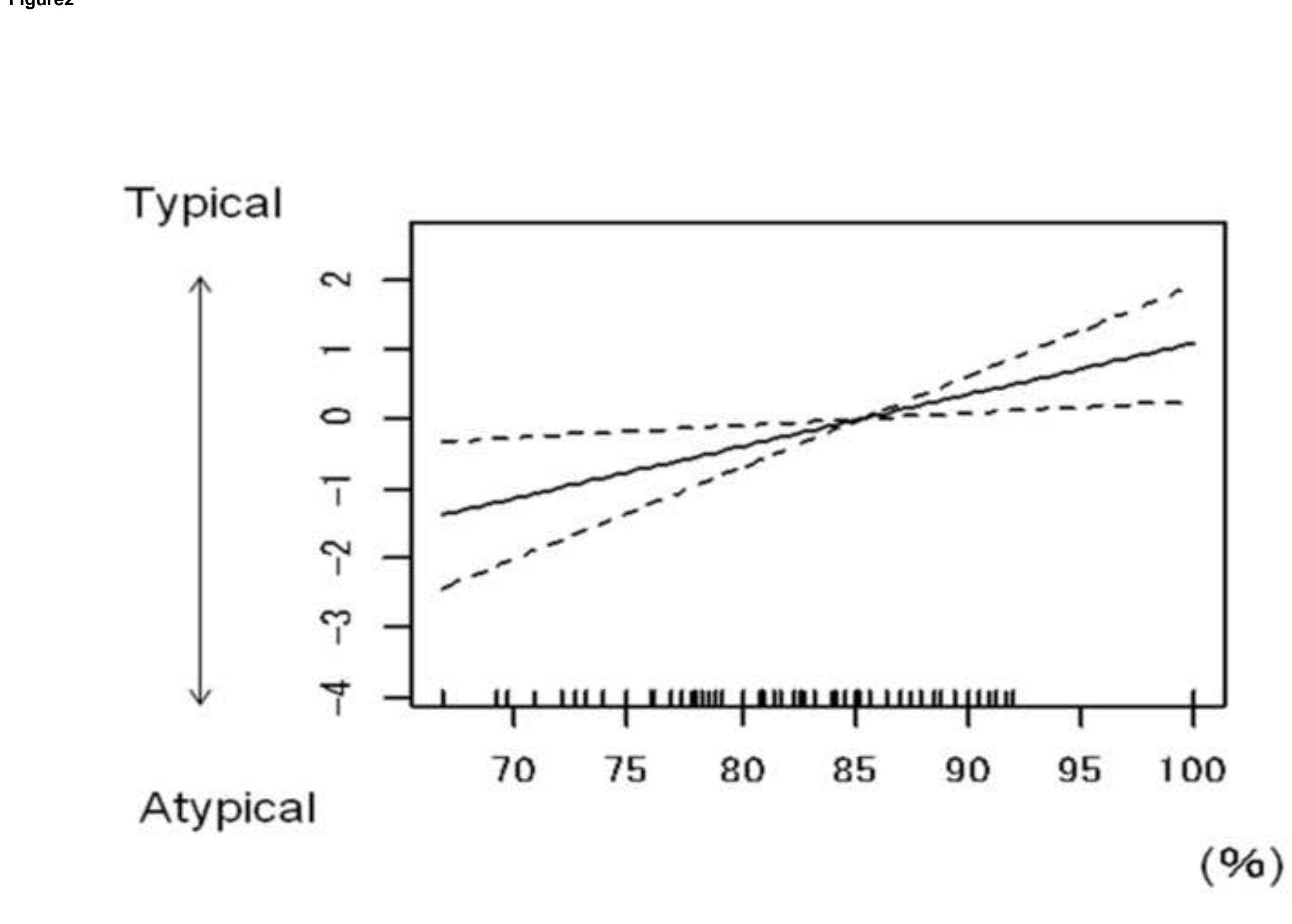

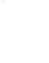

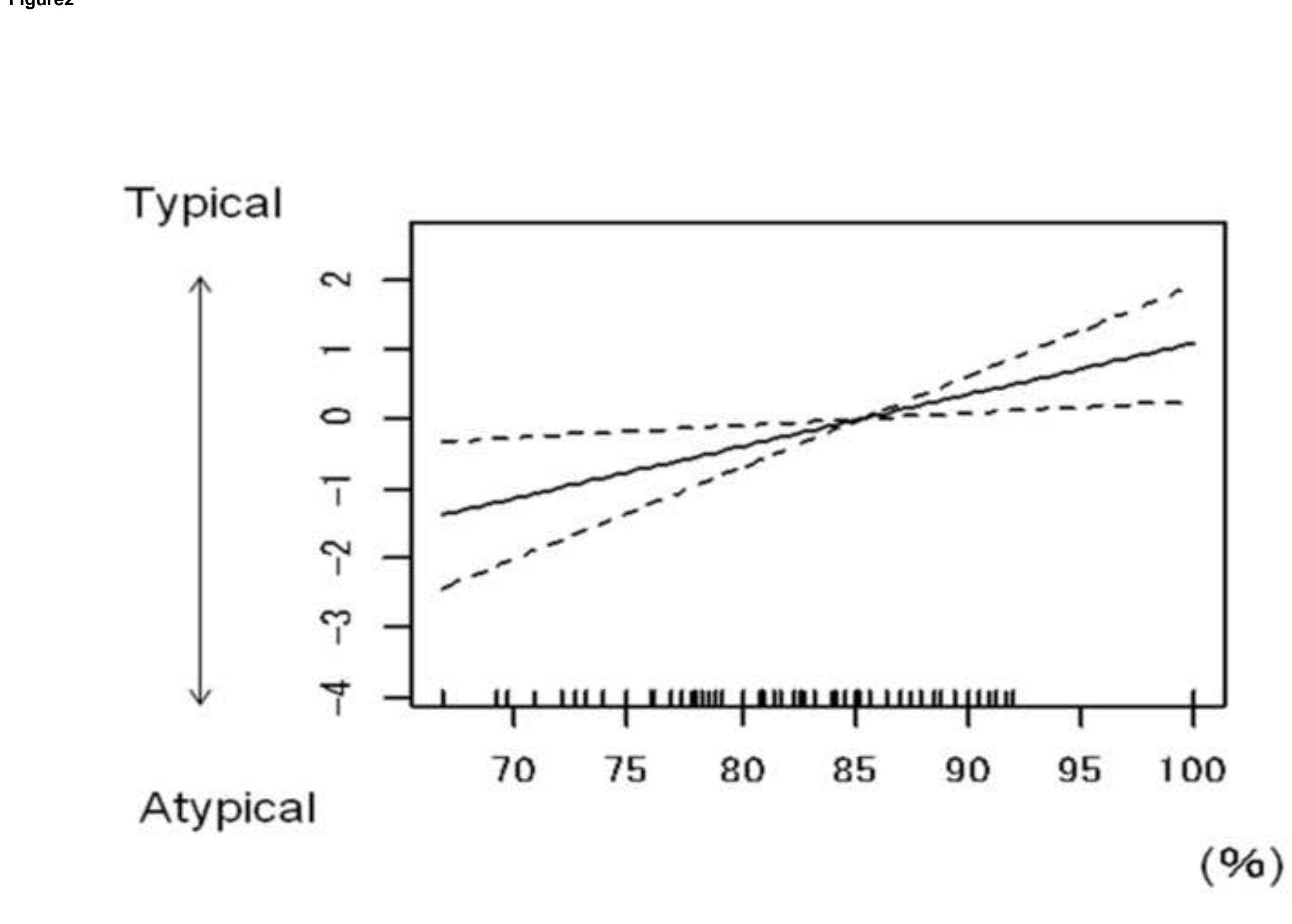

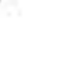




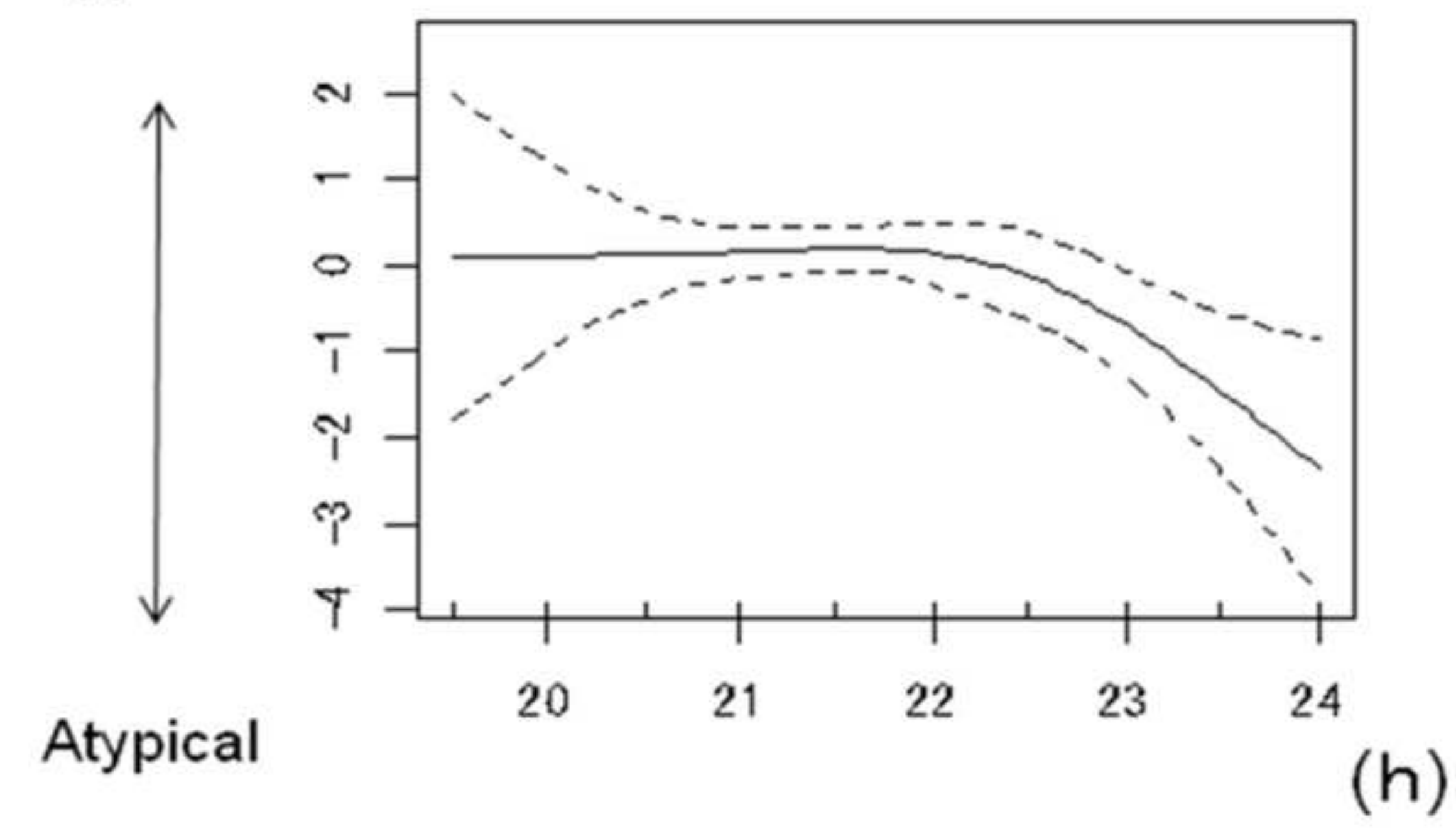

Atypical

\section{Typical}

.




\section{Table 1}

Comparing average values of sleeping-items per development profile of

\section{8-month-old children}

\begin{tabular}{|c|c|c|c|c|}
\hline \multirow[t]{3}{*}{ Variable } & Atypical children & Typical children & \multirow[t]{3}{*}{$\mathrm{P}$} & \multirow[t]{3}{*}{ Value } \\
\hline & $(\mathrm{n}=80)$ & $(\mathrm{n}=300)$ & & \\
\hline & mean $\pm \mathrm{SD}$ & mean $\pm \mathrm{SD}$ & & \\
\hline Bedtime (weekdays), in hour : minutes & $21: 49 \pm 1: 03$ & $21: 30 \pm 0: 53$ & 0.0075 & $* *$ \\
\hline Wake time (weekdays), in hour: minutes & $7: 22 \pm 1: 04$ & $7: 19 \pm 0: 49$ & 0.6938 & \\
\hline Bedtime (saturdays), in hour: minutes & $21: 56 \pm 0: 57$ & $21: 42 \pm 0: 56$ & 0.0559 & \\
\hline Wake time (saturdays), in hour: minutes & $7: 34 \pm 0: 59$ & $7: 36 \pm 54$ & 0.8717 & \\
\hline Bedtime (sundays), in hour : minutes & $21: 56 \pm 1: 42$ & $21: 39 \pm 0: 55$ & 0.029 & $*$ \\
\hline Wake time (sundays), in hour: minutes & $7: 40 \pm 1: 00$ & $7: 43 \pm 0: 57$ & 0.6914 & \\
\hline Morning nap (weekdays), hours & $0.20 \pm 0.49$ & $0.12 \pm 0.37$ & 0.1222 & \\
\hline Afternoon nap (weekdays), hours & $1.84 \pm 0.63$ & $1.76 \pm 0.80$ & 0.4535 & \\
\hline Morning nap (saturdays), hours & $0.19 \pm 0.50$ & $0.08 \pm 0.29$ & 0.0188 & $*$ \\
\hline Afternoon nap (saturdays), houra0 & $1.81 \pm 0.79$ & $1.59 \pm 0.81$ & 0.0358 & $*$ \\
\hline Morning nap (sundays), hours & $0.13 \pm 0.38$ & $0.07 \pm 0.30$ & 0.1546 & \\
\hline Afternoon nap (sundays), hours & $1.55 \pm 0.86$ & $1.43 \pm 0.83$ & 0.2931 & \\
\hline
\end{tabular}




\begin{tabular}{|c|c|c|c|c|}
\hline Time in bed (weekdays), hours & $9.46 \pm 0.93$ & $9.77 \pm 0.78$ & 0.0035 & $* *$ \\
\hline Total sleep time (weekdays), hours & $11.54 \pm 0.87$ & $11.70 \pm 1.08$ & 0.2285 & \\
\hline Sleep efficiency (weekdays), $\%$ & $82.10 \pm 5.83$ & $83.52 \pm 7.41$ & 0.1169 & \\
\hline Time in bed (saturdays), hours & $9.54 \pm 0.97$ & $9.84 \pm 0.83$ & 0.0075 & $* *$ \\
\hline Total sleep time (saturdays), hours & $11.49 \pm 1.13$ & $11.55 \pm 1.06$ & 0.6401 & \\
\hline Sleep efficiency (saturdays), $\%$ & $83.16 \pm 6.48$ & $85.47 \pm 6.72$ & 0.0079 & $* *$ \\
\hline Time in bed (sundays), hours & $9.64 \pm 1.05$ & $9.99 \pm 0.87$ & 0.0025 & $* *$ \\
\hline Total sleep time (sundays), hours & $11.34 \pm 1.23$ & $11.53 \pm 1.08$ & 0.1744 & \\
\hline Sleep efficiency (sundays), $\%$ & $85.23 \pm 6.82$ & $86.70 \pm 8.35$ & 0.1573 & \\
\hline Total nap time (weekdays), hours & $2.02 \pm 0.63$ & $1.89 \pm 0.76$ & 0.1813 & \\
\hline Total nap time (saturdays), hours & $1.97 \pm 0.86$ & $1.67 \pm 0.82$ & 0.006 & $* *$ \\
\hline Total nap time (sundays), hours & $1.68 \pm 0.84$ & $1.58 \pm 1.53$ & 0.5855 & \\
\hline
\end{tabular}

Each value is expressed as mean \pm standard deviation (SD).

* is significantly difficult between typical and atypical children (student $T$ test)

$*$ significntly differnt $(\mathrm{p}<0.05), * *$ significntly differnt $(\mathrm{p}<0.01)$, 


\section{Table 2}

The relation of "development profile of 18-month-old children" and significant sleeping-items by using generalized additive model

\begin{tabular}{|c|c|c|c|c|}
\hline Variable & edf & $\chi^{2}$ & \multicolumn{2}{|c|}{$P$ value } \\
\hline Bed time (Weekdays) & 1.001 & 6.958 & 0.00834 & $* *$ \\
\hline Bed time (saturdays) & 1 & 3.619 & 0.0571 & \\
\hline Bed time（sundays） & 1 & 4.703 & 0.0301 & $*$ \\
\hline number of Afternoon nap (weekdays) & 1 & 4.062 & 0.0439 & $*$ \\
\hline number of Afternoon nap（saturdays） & 1 & 3.323 & 0.0683 & \\
\hline Morning nap（saturdays） & 1.472 & 7.151 & 0.0672 & \\
\hline Afternoon nap（saturdays） & 1 & 4.345 & 0.0371 & $*$ \\
\hline Time in bed (weekdays) & 1.586 & 11.41 & 0.00971 & $* *$ \\
\hline Time in bed (saturdays) & 1.29 & 8.9 & 0.0306 & $*$ \\
\hline Sleep efficiency (saturdays) & 1 & 6.881 & 0.00871 & $* *$ \\
\hline Time in bed (sundays) & 1.765 & 13.38 & 0.00388 & $* *$ \\
\hline Total nap time (saturdays) & 2.701 & 10.81 & 0.0128 & * \\
\hline
\end{tabular}

$*$ Significntly differnt $(\mathrm{p}<0.05), * *$ Significntly $\operatorname{differnt}(\mathrm{p}<0.01)$,

edf, equivalent degree of freedom 
Table 3.

Risk factors for adverse sleep times in infants

\begin{tabular}{|c|c|c|c|c|}
\hline parameter & estimate & SE & Wald $\chi 2$ & $\mathrm{Pr}>\mathrm{ChiSq}$ \\
\hline intercept & -10.4024 & 7.09 & 2.15 & 0.14 \\
\hline Discipline $(<19)$ & -0.7264 & 0.19 & 14.07 & 0.0002 \\
\hline Discipline $(>19)$ & 0.2095 & 0.15 & 2.03 & 0.15 \\
\hline Expressive language & -0.2804 & 0.08 & 11.94 & 0.0006 \\
\hline Receptive language & -0.2370 & 0.07 & 10.93 & 0.0009 \\
\hline Head circumference & 0.3559 & 0.14 & 6.88 & 0.0087 \\
\hline Sleep efficiency (saturdays) & -0.0760 & 0.03 & 7.15 & 0.0075 \\
\hline Concept & 0.2332 & 0.07 & 10.56 & 0.0012 \\
\hline Sleep onset(week day) $>22$ & 1.0796 & 0.36 & 8.83 & 0.0030 \\
\hline Approach/withdrawal to new situation & 0.3874 & 0.14 & 7.92 & 0.0049 \\
\hline \multicolumn{5}{|l|}{ Approach/withdrawal to new } \\
\hline & -0.0112 & 0.003 & 9.27 & 0.0023 \\
\hline situation(square) & & & & \\
\hline
\end{tabular}

SE, standard error 